\title{
Characterization of novel intramedullary nailing method for treating femoral shaft fracture through finite element analysis
}

\author{
YUNWEI CUI $^{1}$, WENZHAO XING ${ }^{2}$, ZHENHUA PAN $^{2}$, ZHIGANG KONG $^{2}$, \\ LIANG SUN ${ }^{2}$, LEI SUN ${ }^{2}$, XIAODONG CHENG ${ }^{1}$ and CHANGCHENG LIU ${ }^{2}$ \\ ${ }^{1}$ Key Laboratory of Biomechanics of Hebei Province; ${ }^{2}$ Department of Orthopaedic Surgery, \\ The Third Hospital of Hebei Medical University, Shijiazhuang, Hebei 050051, P.R. China
}

Received March 8, 2019; Accepted July 12, 2019

DOI: $10.3892 /$ etm.2020.8763

\begin{abstract}
Femoral shaft fracture is one of the most common types of fracture encountered in the clinic. For certain complex femoral shaft fractures, the traditional intramedullary nail may not provide sufficient stability. Therefore, novel intramedullary nail systems are required. The femur 3D model and the internal fixation model were designed using Mimics 17.0 (Materialise), Geomagic Studio 2012 (Raindrop) and Solidwork 2016 (Dassault) software. The validity of the models was verified through comparison with previous data in silico. To further simulate the comminuted femoral shaft fracture, the novel and traditional type of intramedullary nail system were included in the finite element analysis with the software. The displacement and stress distribution of the two internal fixations were compared using Abaqus 6.14 (Dassault) software. The effectiveness of the model was verified. The stress at the fixed end of the novel intramedullary nail system was greater than that at the fixed end of the traditional intramedullary nail system. However, the displacement of the novel intramedullary nail method was smaller than that of the traditional intramedullary nail. The novel intramedullary nail system features good stability and stress stimulation at the broken end, which is beneficial for bone healing. The present study may provide a theoretical basis for the selection of a means of internal fixation in the clinic.
\end{abstract}

\section{Introduction}

The femur is the longest bone and the major weight-bearing bone of the lower limbs in the human body. Femoral shaft fracture is one of the most common fractures in the clinic, accounting for $18.5 \%$ of limb fractures and $6 \%$ of total bone fractures (1). Femoral shaft fracture refers to the fracture between the femoral

Correspondence to: Dr Changcheng Liu, Department of Orthopaedic Surgery, The Third Hospital of Hebei Medical University, 139 Ziqiang Road, Shijiazhuang, Hebei 050051, P.R. China

E-mail: liu_changcheng84@outlook.com

Key words: femoral shaft fracture, intramedullary nail, displacement, stress, finite element condyle and femur. Adult femoral shaft fractures are frequently caused by high-energy injuries and post-fracture complications are common. If it not treated properly, complications may seriously affect the quality of life of patients and may even be life-threatening (2).

With the continuous improvement of internal fixation materials, surgical methods and changes in concepts of fracture treatment methods, adult femoral shaft fractures tend to be treated with surgical treatment combined with internal fixation. Since the 19th century, bone plate and intramedullary nail have been gradually used in the treatment of femoral shaft fractures. Owing to its compatibility with the bone mechanical properties and its ability to align the bone fragments, intramedullary nail is the first choice of treatment for femoral shaft fractures (3).

However, for complex femoral shaft fractures, including Winquist III and IV femoral shaft fractures, traditional intramedullary nails may still not provide sufficiently reliable stability. Furthermore, non-surgical treatment is associated with a long bed rest time and common complications thereof. In order to solve this problem, a novel intramedullary nail system was developed in the present study. The major characteristics of the novel intramedullary nail are that the nail body are slotted and the elastic modulus of the nail is reduced after rotation. The novel intramedullary nail has two advantages when compared with traditional nails: i) Fragments may be fixed by a Kirschner wire at the grooved site and ii) elasticity may be reduced to accommodate different bone fractures, particularly in elderly patients with osteoporosis.

However, as the human body is a complex mechanical structure, it is challenging to simulate human mechanics using biomechanical experiments. The finite element method has been mainly applied to biomechanical research in the medical field. As the finite element method may simulate the biomechanical behavior of the human body, it was used as an effective method to understand its mechanical characteristics. Its effectiveness and superiority have been fully demonstrated in basic experiments and clinical applications (4-6). The finite element method may divide the continuous elastic bodies into a finite number of units, synthesize the research subject and study the properties of each unit by using the finite element software in the computer.

In the present study, the finite element method was employed to analyze and evaluate the biomechanical status of novel and traditional intramedullary nails prior to and after 
femoral shaft fracture healing. The present study aimed to provide a biomechanical basis for surgeons to select a more effective fixation method with satisfactory therapeutic effects.

\section{Materials and methods}

Volunteer and femoral scan. A 30-year-old healthy male with a body height of $172 \mathrm{~cm}$ and a body weight of $75 \mathrm{~kg}$ was selected as a volunteer. The subject had no history of femoral fracture or trauma, and femoral lesions and injuries were also ruled out by X-ray imaging. The spiral axis scan of the bilateral femurs was performed by using a CT scanner (Optima CT 660; GE Healthcare) from top to bottom using the scanning condition of $120 \mathrm{KV}, 125 \mathrm{~mA}$ and a layer thickness of $1 \mathrm{~mm}$. The above machine had an advanced image post-processing and camera function.

After the original data were interpolated and enlarged on the machine, a continuous picture of the layer thickness was obtained, and was burned to the optical disc in the international standard dicom format. The study was approved by the Ethics Committee of The Third Hospital of Hebei Medical University (Shijiazhuang, China). The internal fixation model was built using Solidwork 2016 software (Dassault); the novel intramedullary nail system was designed by the Third Hospital of Hebei Medical University (patent number, ZL201721394941.3).

Construction of normal femur 3D model. The femur computed tomography (CT) image (dicom format) was imported into the Mimics 17.0 software (Materialise) to segment bonefrom the entire model bythresholding; the images were subsequently selectively segmented using 'split-mask' according to the anatomical structure. Finally, a geometric model of the femur was established through regional expansion, smoothing all parts of the femur, filling small gaps, and making the outer contour of the femur smooth and continuous.

The constructed femur information was saved in an STL format file, and the STL format file was repaired and optimized by reverse engineering software Geomagic Studio 2012 (Raindrop). In brief, the non-characteristic masses and indentations on the surface of the model were removed and the surface was smoothed. Furthermore, non-characteristic high curvature and the generation of self-intersecting faces were prevented. The smooth surface of the model was used to fit the triangular surface of the model surface; the femoral cortical bone and cancellous bone were distinguished by using the offset function. At last, a continuous curved surface model was generated.

Boundary and load information. It was assumed that the fracture surface was completely broken and in a contact state. The friction coefficient was 0.2 according to a previous study (7). The femur was subjected to numerous loads, including muscle force, joint force and dynamic impact force. In view of numerous uncertainties, including the number and direction of load during finite element simulation of muscle loading, it was relatively complex to accurately simulate the loading conditions in the dynamic state (8). The simple model method of Duda et al (9) was used in the present study. To simulate the load of the femur while standing and walking, analysis ofthe static mechanics of the model was performed. The condition was that the distal knee joint had a degree of freedom of 0 and the distal nodes have a displacement
Table I. Properties of materials included in the model.

\begin{tabular}{lcc}
\hline $\begin{array}{l}\text { Material } \\
\text { name }\end{array}$ & $\begin{array}{c}\text { Modulus of } \\
\text { elasticity (MPa) }\end{array}$ & $\begin{array}{c}\text { Poisson's } \\
\text { ratio }\end{array}$ \\
\hline Cortical bone & 16,800 & 0.29 \\
Cancellous bone & 260 & 0.20 \\
Traditional intramedullary rod & 110,000 & 0.30 \\
Novel intramedullary rod & 110,000 & 0.30 \\
\hline
\end{tabular}

of 0 on the $\mathrm{X}, \mathrm{Y}$ and $\mathrm{Z}$ axes. Furthermore, a load of $700 \mathrm{~N}$ along the direction of the force line at the outer surface of the contact area between the femoral head and the acetabulum was applied.

According to Duda et al (9), the intact femur received a torque of $\sim 0.04$ body weight meters (BWM) during the gait cycle. However, three weeks after the intramedullary nailing of the femoral shaft fracture, the maximum torque of the femur was $0.01 \mathrm{BWM}(10)$. Therefore, based on the load of $700 \mathrm{~N}$, the maximum torque of the femur was $7-28 \mathrm{Nm}$. Therefore, in the torsion analysis under exclusion of the specific effects of each muscle group, the boundary load condition was fixation on the femoral condyles and a 7-Nm external rotation torque was applied at the proximal end of the femur along the same axis as compressive loading.

\section{Results}

Construction and properties of the normal femur $3 D$ model. In general, biological tissue belongs to an anisotropic non-linear body. The application of bone finite element analysis in the field of biomechanics is mostly based on the isotropic, homogeneous continuous linear elastomer. Therefore, the model utilized in the present study assumed that the femoral bone is an isotropic, homogeneous linear elastic material, which consists of cortical bone, cancellous bone and internal fixation (Table I), and also referred to the data reported by previous studies $(11,12)$. Only two materials of bone and internal fixation were considered in the model, and muscle and tendon stress were simplified, regardless of the friction between the joints and cartilage. A femoral geometric model was established by using Mimics 17.0 (Materialise) and Geomagic Studio 2012 (Raindrop) software based on the CT image of the femur (Fig. 1).

Construction of two different internal fixation models for comminuted fracture of the femoral shaft. The traditional intramedullary nail system (Fig. 2) was based on the intramedullary nail (diameter, $10 \mathrm{~mm}$; length, $380 \mathrm{~mm}$ ) produced by Double Medical Technology Co., Ltd. (Fig. 2Aa). Solidwork 2016 (Dassault) is a software for simulation design and has been applied in numerous fields, including aviation and machinery, where the modeling results were reliable (6). The internal fixation model was built using the Solidwork 2016 (Dassault) software, a novelintramedullary nail system designed in The Third Hospital of Hebei Medical University in 2017 (patent no: ZL201721394941.3; Fig. 2Bb). The geometric structure and the specific parameters of the novel intramedullary nailing system are provided in Fig. S1. It is designed as a hollow nail body, including the head, body and tail of the nail. The nail 

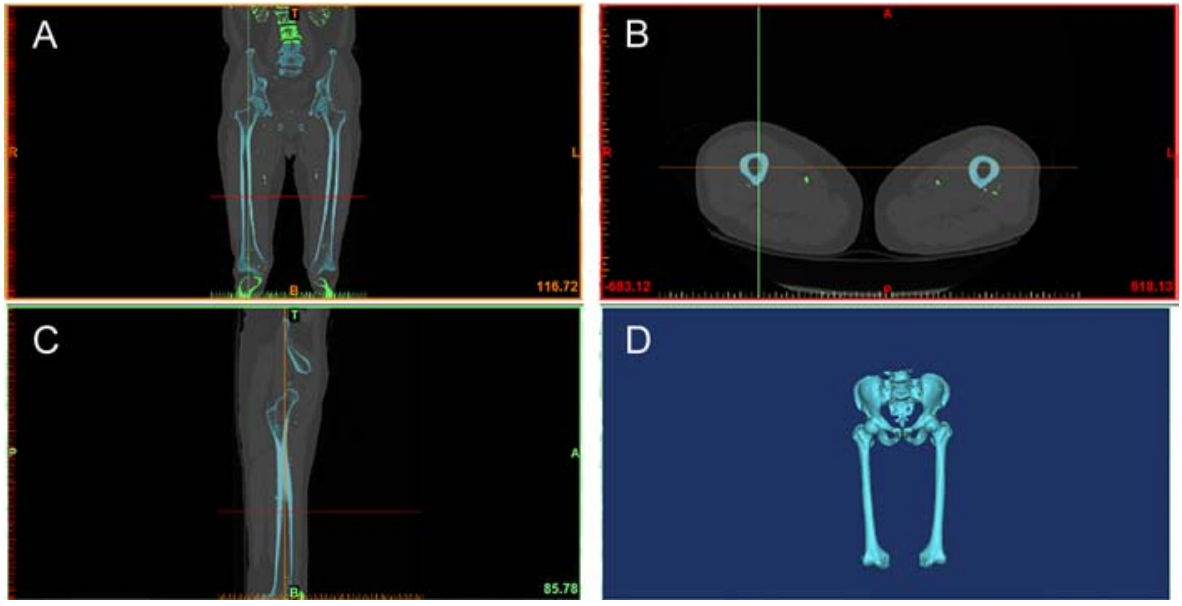

Figure 1. Establishment of femoral geometric model using the Mimics 17.0 (Materialise), Geomagic Studio 2012 (Raindrop) and Solidwork 2016 (Dassault) softwares. (A) Coronal view of the CT data. (B) Cross-sectional view of the CT data. (C) Sagittal view of the CT data. (D) Three-dimensional view of the CT data.

A
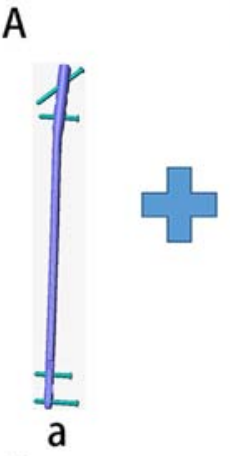

B

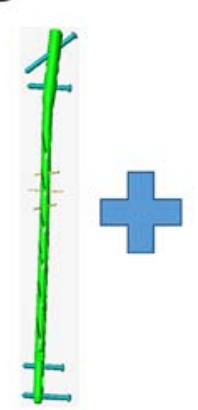

a
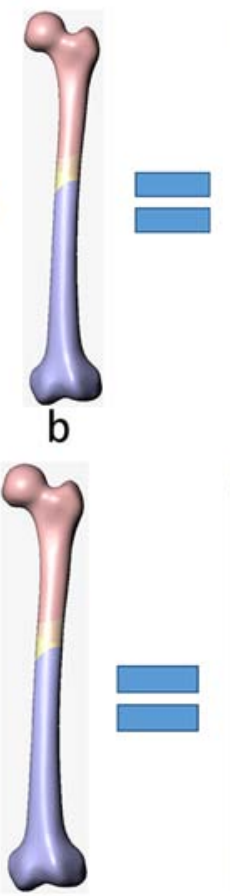

b

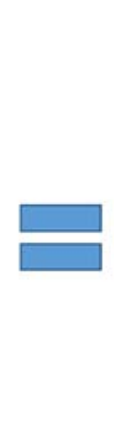

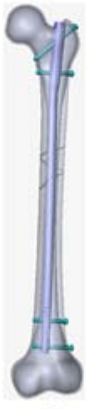

C

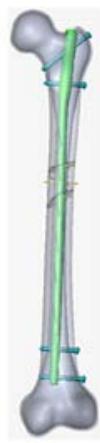

C

Figure 2. Composition of the traditional and novel intramedullary nail internal fixation model. (A) Composition of the traditional and intramedullary nail internal fixation model. (a) the internal fixation model was built using Solidworks software. The traditional intramedullary nail system built in this experiment is based on the intramedullary nail (diameter, $10 \mathrm{~mm}$; length, $380 \mathrm{~mm}$ ) produced by Dabo Technology Co., Ltd. (b) The Third Hospital of Hebei Medical University designed the prototype in 2017. (c) The femoral solid model generated using Geomagic Studio was imported into the design software Solidworks in IGES format to simulate the femoral shaft fracture. (B) Composition of the novel intramedullary nail internal fixation model. (a) Novel intramedullary nail system. (b) Femoral shaft fracture model. (c) Novel intramedullary nail internal fixation model. IGES, Initial Graphics Exchange Specification.

body includes a slotted section and a corresponding connecting section. The upper ring of the side wall of each grooved section has several grooves connected to the mouth of the nail body, which are spirally arranged along the extension direction of the nail body.

A

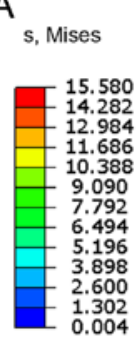

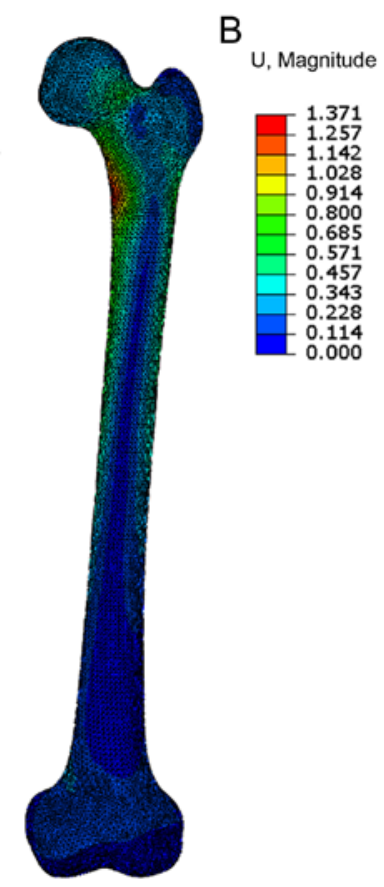

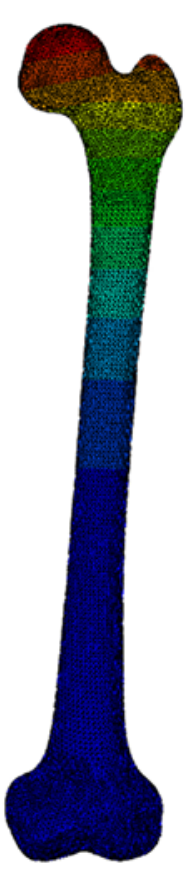

Figure 3. Finite element analysis results of a complete femoral model under a $700 \mathrm{~N}$ load, generated using the Abaqus 6.14 software. (A) Stress cloud map; (B) displacement cloud map.

The femoral solid model in IGES format generated by Geomagic Studio 2012 (Raindrop) was imported into the design software Solidwork 2016 (Dassault) to simulate femoral shaft fractures (Fig. 2Ac and Bc). Combined with the above internal fixation model, two different internal fixation femoral shaft fractures in three-dimensional finite element models were established (Fig. 2Ad and Be).

Finite element analysis of the complete femur model and comminuted femoral shaft fracture. After verifying the validity of the above traditional and novel femoral finite element models (Fig. 2A and B), the femoral comminuted fractures were fixed with the traditional and novel intramedullary nail systems. Subsequently, the finite element method 


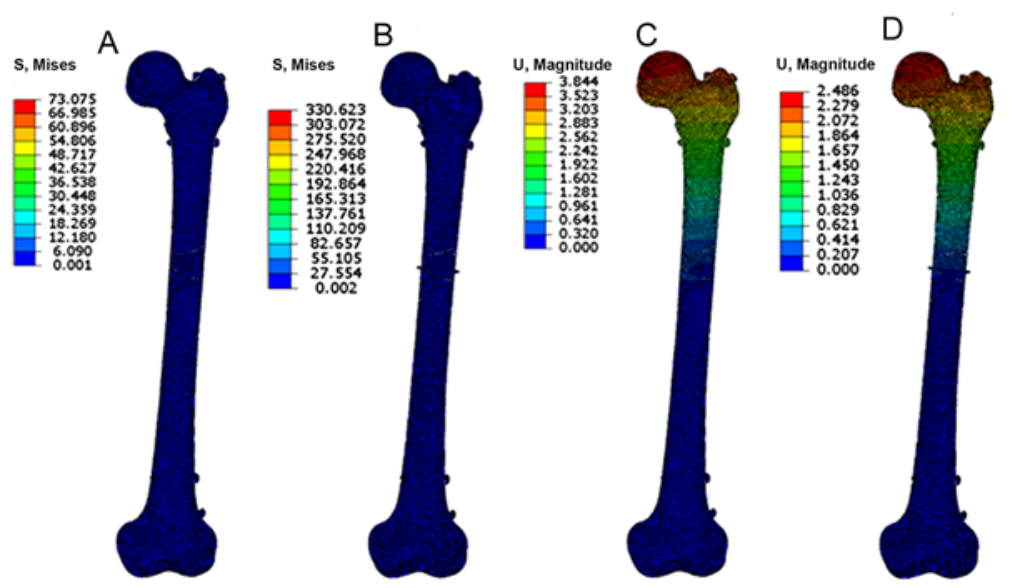

Figure 4. Compression analysis results of a complete femoral model under a $700 \mathrm{~N}$ load, generated using the Abaqus 6.14 software. Compressed stress cloud map of (A) traditional and (B) novel intramedullary nail compression prior to healing. Compressed displacement cloud map of (C) traditional and (D) novel intramedullary nail compression prior to healing.
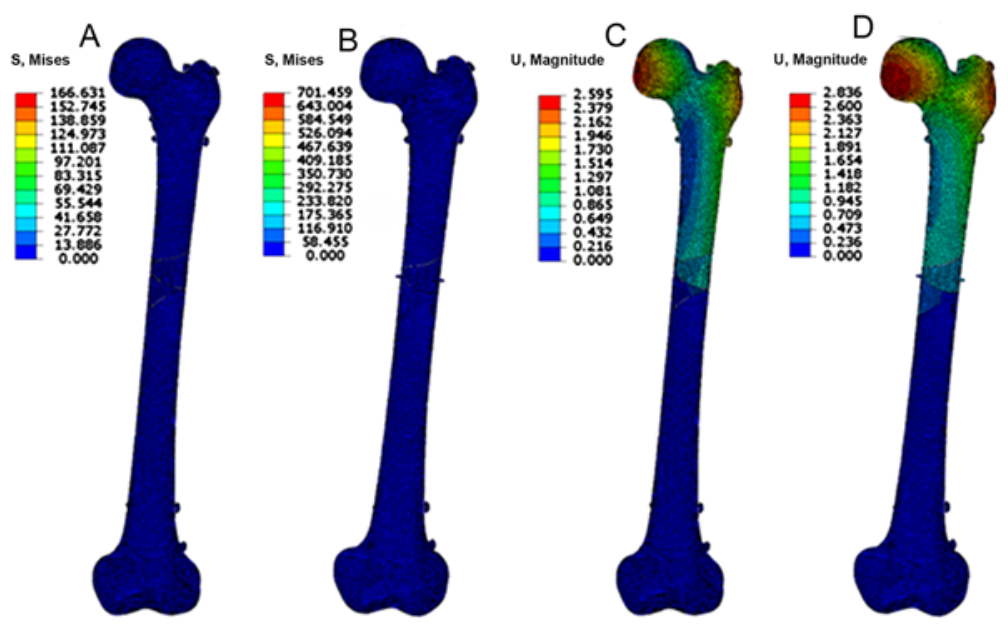

Figure 5. Torsional analysis results of a complete femoral model under a $700 \mathrm{~N}$ load, generated using the Abaqus 6.14 software. Torsional stress cloud map of (A) traditional and (B) novel intramedullary nail compression prior to healing. Torsional displacement cloud map of (C) traditional and (D) novel intramedullary nail compression prior to healing.

was used to analyze a complete femoral model under a 700-N load. The stress cloud map (Fig. 3A) and the displacement cloud map (Fig. 3B) were also provided. The stress was mainly distributed in the middle and lower part of the femur, around the femoral neck and the large and small trochanter. There was also an obvious stress concentration at the small trochanter of the femur (Figs. 4-6), prior to healing as well as after healing.

Under the compressive load, the maximum stresses, the maximum displacements, broken bone stresses and broken bone displacements among the traditional and the novel intramedullary nail fixation prior to healing, the traditional and novel intramedullary nailing after healing, and the complete femur were also provided in Table II. Under torsional load, the traditional and novel intramedullary nail fixations prior to healing were also compared (Table III).

When subjected to compressive or torsional load prior to complete healing, the stability of the novel IM nail fixation was greater compared with that of the traditional IM nail fixation, as indicated by the smaller resulting maximum fracture site displacement (Table III). The novel IM nail shares more loads as indicated by the higher maximum stress in the novel intramedullary nail group compared with the traditional IM nail group (Table III). The fragment stress in the novel IM nail under both loading conditions were higher because of the higher load concentration around Kirschner wires in the fragment.

It was suggested that the novel intramedullary nail fixation system was superior compared with traditional intramedullary nail fixation systems with regards to maximum stress and displacement, which stimulates bone growth.

\section{Discussion}

Femoral shaft fracture is a common type of fracture encountered in the clinic. At present, the traditional intramedullary nail may not provide sufficiently reliable results for partial complex femoral shaft fractures. The present study mainly discussed the partial stress distribution of the femur after internal fixation with the novel or traditional nail under various 
Table II. Comparison of bone stress and displacement in different models of nail fixation under compressive load prior to and after healing.

\begin{tabular}{lcccc}
\hline Item & $\begin{array}{c}\text { Maximum } \\
\text { stress (MPa) }\end{array}$ & $\begin{array}{c}\text { Maximum } \\
\text { displacement (mm) }\end{array}$ & $\begin{array}{c}\text { Bone fragment } \\
\text { stress (MPa) }\end{array}$ & $\begin{array}{c}\text { Bone fragment } \\
\text { displacement (mm) }\end{array}$ \\
\hline Traditional intramedullary nail fixation prior to healing & 73.1 & 3.84 & 5.30 & 0.691 \\
Novel intramedullary nail fixation prior to healing & 330 & 2.49 & 17.5 & 0.343 \\
Traditional intramedullary nail fixation after healing & 38.8 & 1.21 & & \\
Novel intramedullary nail fixation after healing & 81.0 & 1.28 & & \\
Complete femur & 15.6 & 1.37 & & \\
\hline
\end{tabular}

Table III. Comparison of bone stress and displacement in the traditional and novel intramedullary nail fixation prior to healing under torsional load.

\begin{tabular}{lcccc}
\hline Item & $\begin{array}{c}\text { Maximum } \\
\text { stress (MPa) }\end{array}$ & $\begin{array}{c}\text { Maximum } \\
\text { displacement (mm) }\end{array}$ & $\begin{array}{c}\text { Bone fragment } \\
\text { stress (MPa) }\end{array}$ & $\begin{array}{c}\text { Bone fragment } \\
\text { displacement (mm) }\end{array}$ \\
\hline Traditional intramedullary nail fixation prior to healing & 166 & 2.59 & 0.749 & 0.767 \\
Novel intramedullary nail fixation prior to healing & 701 & 2.84 & 39.7 & 0.754 \\
\hline
\end{tabular}
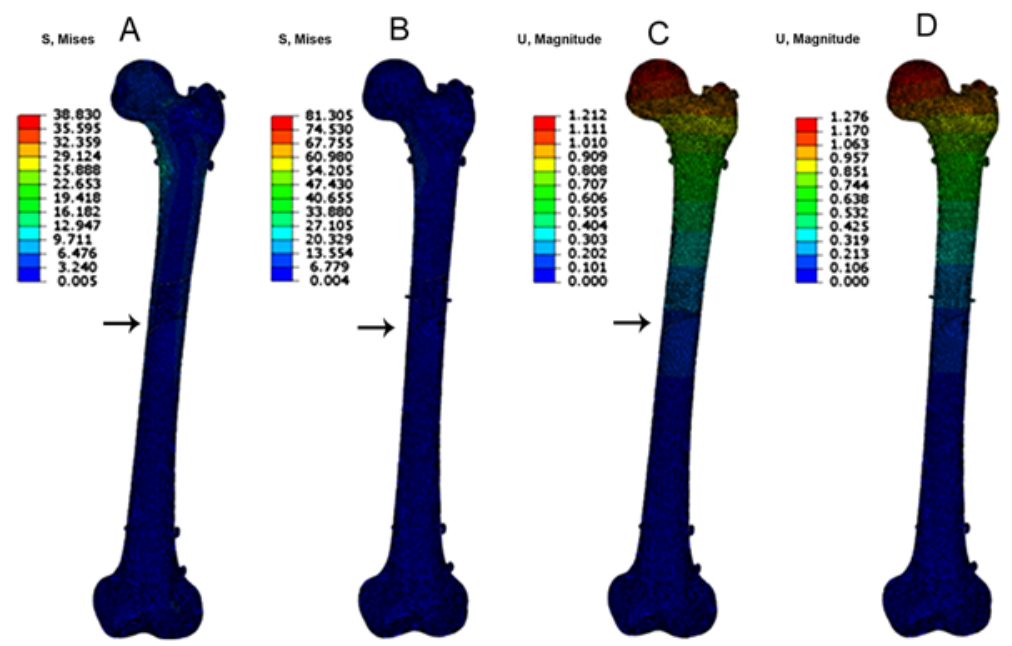

Figure 6. Compression analysis results of a complete femoral model after healing, generated using the Abaqus 6.14 software. Compressed stress cloud map of (A) traditional and (B) novel intramedullary nail compression after healing. Compressed displacement cloud map of (C) traditional and (D) novel intramedullary nail compression after healing. Black arrows indicate the location of the fracture site.

static loading conditions. The major difference between the novel and the traditional intramedullary nails is that the body of the novel nail includes a plurality of slots, and the slot is spirally arranged along the extending direction of the body portion. It may adjust the curvature of the local part of the nail body to match the medullary cavity of different patients, fix the bone fragments and shorten the healing time of the fracture.

Intramedullary nail acts as a load sharing device with peri-fracture site compared to the load bearing metallic bone plate. In the early post-fracture period, the novel intramedullary nail facilitates greater force transmission through the fracture site, which is beneficial for fracture healing. The stress of the intramedullary nail after fracture healing is less than the stress endured by the healthy femur, which indicates that force after healing was mainly performed by the femur. There was also an obvious stress concentration at the small trochanter of the femur prior to and after healing. This was consistent with previous studies $(13,14)$, suggesting the successful establishment of an effective and reliable model in the present study. The displacement of the femur under load was small, indicating that the femur has a large stiffness. In the early stage of healing, the femur should not be subjected to a large stress, and as the affected area heals, the femur also bears more force. The novel intramedullary nail fixation system is able to eliminate the stress shielding effect along the long axis of the femur and allows for axial compression of the fracture site. The fretting 
continuous axial load of the fracture site may promote fracture healing (15).

In conclusion, the present study indicated that appropriate pressure at the fracture end eliminates the gap at the fracture end. This improves the static friction between the broken femurs, which increases the fixing strength and mechanical stability, and is also beneficial for bone growth and crawling replacement. The novel intramedullary nail fixation reduced stress shielding and promoted stress transmission. As further demonstrated in another study, compressive stress-mediated changes may effectively stimulate cell differentiation, which may promote fracture healing and stimulate bone metabolism during the fixed pressurization (16). Finally, displacement of the femoral head and the broken end in the novel intramedullary nail fixation system were far less than those in the traditional intramedullary nail system, providing a significantly improved fixation effect of the intramedullary nail. These improvements in stability have a beneficial role in promoting fracture healing.

\section{Acknowledgements}

Not applicable.

\section{Funding}

No funding was received.

\section{Availability of data and materials}

The datasets used and analyzed during the present study are available from the corresponding author on reasonable request.

\section{Authors' contributions}

YWC was responsible to the study concepts, manuscript preparation and editing; WZX was responsible for the clinical studies; ZHP was responsible for the definition of intellectual content and literature research; ZGK was in charge of the data acquisition; LiS was responsible for the statistical analysis; LeS was responsible to the data analysis; XDC was responsible to the experimental studies; CCL was the guarantor of integrity of the entire study, study design and manuscript review. All authors approved the final version of this manuscript.

\section{Ethics approval and consent to participate}

The study was approved by the Third Hospital of Hebei Medical University (Shijiazhuang, China). Informed consent of images publication and data usage were also obtained (patent number, ZL201721394941.3).

\section{Patient consent for publication}

Not applicable.

\section{Competing interests}

The authors declare that they have no competing interests.

\section{References}

1. Fakhry SM, Rutledge R, Dahners LE and Kessler D: Incidence, management and outcome of femoral shaft fracture: A statewide population-based analysis of 2805 adult patients in a rural state. J Trauma 37: 255-260; discussion 260-261, 1994.

2. Morishige M, Muramatsu K, Tominaga Y, Hashimoto T and Taguchi T: Surgical treatment of metastatic femoral fractures: Achieving an improved quality of life for cancer patients. Anticancer Res 35: 427-432, 2015.

3. Cheung G, Zalzal P, Bhandari M, Spelt JK and Papini M: Finite element analysis of a femoral retrograde intramedullary nail subject to gait loading. Med Eng Phys 26: 93-108, 2004.

4. Abueidda DW, Dalaq AS, Abu Al-Rub RK and Younes HA: Finite element predictions of effective multifunctional properties of interpenetrating phase composites with novel triply periodic solid shell architectured reinforcements. Int J Mech Sci 92: 80-89, 2015.

5. Ramos Verri F, Santiago Junior JF, de Faria Almeida DA, de Oliveira GB, de Souza Batista VE, Marques Honório H, Noritomi PY and Pellizzer EP: Biomechanical influence of crown-to-implant ratio on stress distribution over internal hexagon short implant: 3-D finite element analysis with statistical test. J Biomech 48: 138-145, 2015.

6. Sternheim A, Giladi O, Gortzak Y, Drexler M, Salai M, Trabelsi N, Milgrom C and Yosibash Z: Pathological fracture risk assessment in patients with femoral metastases using CT-based finite element methods. A retrospective clinical study. Bone 110: 215-220, 2018.

7. Robinson PS, Placide R, Soslowsky LJ and Born CT: Mechanical strength of repairs of the hip piriformis tendon. J Arthroplasty 19: 204-210, 2004.

8. Paul JP: Stress and strain distribution within the intact femur: Compression or bending? by Taylor et al. Med Eng Phys 19: 97, 99-100, 1997.

9. Duda GN, Schneider E and Chao EY: Internal forces and moments in the femur during walking. J Biomech 30: 933-941, 1997.

10. Schneider E, Michel MC, Genge M, Zuber K, Ganz R and Perren SM: Loads acting in an intramedullary nail during fracture healing in the human femur. J Biomech 34: 849-857, 2001.

11. Perez JV, Warwick DJ, Case CP and Bannister GC: Death after proximal femoral fracture-an autopsy study. Injury 26: 237-240, 1995.

12. Goosen JH, Mulder MC, Bongers KJ and Verheyen CC: High revision rate after treatment of femoral neck fractures with an optionally (un) cemented stem. Arch Orthop Trauma Surg 129: 801-805, 2009.

13. Valliappan S, Svensson NL and Wood RD: Three dimensional stress analysis of the human femur. ComputBiol Med 7: 253-264, 1977.

14. Couteau B, Hobatho MC, Darmana R, Brignola JC and Arlaud JY: Finite element modelling of the vibrational behaviour of the human femur using CT-based individualized geometrical and material properties. J Biomech 31: 383-386, 1998.

15. Bottlang M, Tsai S, Bliven EK, von Rechenberg B, Klein K, Augat P, Henschel J, Fitzpatrick DC and Madey SM: Dynamic stabilization with active locking plates delivers faster, stronger, and more symmetric fracture-healing. J Bone Joint Surg Am 98: 466, 2016.

16. Cole JD, Blum DA and Ansel LJ: Outcome after fixation of unstable posterior pelvic ring injuries. Clin Orthop Relat Res: 160-179, 1996.

This work is licensed under a Creative Commons Attribution-NonCommercial-NoDerivatives 4.0 International (CC BY-NC-ND 4.0) License. 